\title{
Antibacterial Surfaces, Thin Films, and Nanostructured Coatings
}

\author{
Daniele Valerini
}

Citation: Valerini, D. Antibacterial Surfaces, Thin Films, and

Nanostructured Coatings. Coatings 2021, 11, 556. https://doi.org/ 10.3390/coatings11050556

Received: 6 April 2021

Accepted: 4 May 2021

Published: 8 May 2021

Publisher's Note: MDPI stays neutral with regard to jurisdictional claims in published maps and institutional affiliations.

Copyright: (C) 2021 by the author. Licensee MDPI, Basel, Switzerland. This article is an open access article distributed under the terms and conditions of the Creative Commons Attribution (CC BY) license (https:// creativecommons.org/licenses/by/ $4.0 /)$.
ENEA-Italian National Agency for New Technologies, Energy and Sustainable Economic Development, SSPT-PROMAS-MATAS, S.S. 7 Appia, km 706, 72100 Brindisi, Italy; daniele.valerini@enea.it

Antibacterial surfaces can play a key role in a great number of everyday applications, spanning from biomedical purposes (medical devices, protection equipment, surgery tools, human implants, etc.) to usages for food and beverages (e.g., packaging). Such surfaces are fundamental to prevent the occurrence and diffusion of clinical infections and foodborne diseases, or to preserve the quality of the packaged content.

Different approaches can be pursued to confer antimicrobial properties to a given surface, such as the incorporation of antibacterial agents within the material surface or their deposition as coating films. Several organic (enzymes, natural extracts, etc.) and inorganic (metals, oxides, etc.) antibacterial agents, each with their own peculiar characteristics, are continuously studied and tested, seeking for ever-improved performance. In particular, in recent decades, new and ever more efficient materials have been experimented and effectively used in the aforementioned applications, especially after the enormous advances in nanotechnologies.

At the same time, together with the antimicrobial performance, for such kinds of applications, other essential aspects should be considered, such as the enhancement of other materials properties (mechanical, optical, wettability, etc.), as well as human safety issues, biocompatibility and environmental aspects.

In this framework, this Special Issue was aimed to collect original research works and reviews dealing with antibacterial surfaces and related aspects, from advances in materials and surface engineering, to characterization and functional properties, toxicity/safety for human health, and environmental aspects.

This Special Issue includes nine research papers and one review, relating to various strategies aimed at contrasting bacterial proliferation on surfaces or studying the biocompatibility of materials for many different applications.

In the first research work [1], different amounts of silica $\left(\mathrm{SiO}_{2}\right)$ nanoparticles were added into Poly(methyl methacrylate) (PMMA) to fabricate a reinforced nanocomposite material with improved mechanical properties, evaluating its cytotoxic effects with the aim to get a biocompatible material with possible applications in dentures.

A material widely employed in implants is titanium, where it needs to exhibit biocompatibility and antimicrobial properties. In work [2], titanium foils were coated with a blend made of chitosan (CS) and zinc-coated halloysite nanotubes (ZnHNTs) loaded with gentamicin sulfate. Such composite coating was tested in order to get a biocompatible material able to provide antimicrobial action against Staphylococcus aureus thanks to the release of metal ions and antibiotic gentamicin.

Similarly, another composite material, which could deliver possible controlled release of antimicrobial agents, was studied in the work presented in paper [3]. Here, a photoelectrochemical reduction process was employed to synthesize silver nanoparticles (Ag NPs) in a hydroxypropyl methylcellulose (HPMC) matrix, whose bactericidal effects were tested against Escherichia coli and Staphylococcus epidermidis species. This system was then expected to represent a promising material for the controlled release of antibacterial Ag NPs through the slow dissolution of HPMC in water. 
With the aim to prevent formation of biofilms of multi-drug resistant pathogens, the authors of paper [4] developed zinc oxide nanoparticles (ZnO NPs) functionalized with extracellular polysaccharide xanthan gum $(X G)$ by a green route. The quorum sensing inhibitory activity of this nanocomposite was evaluated against Gram-negative pathogens Chromobacterium violaceum and Serratia marcescens, demonstrating a significant inhibition of biofilm formation.

With the same aim to develop new materials against antimicrobial-resistant (AMR) bacteria, in the work presented in paper [5] different metal ions ( $\mathrm{Ag}, \mathrm{Au}, \mathrm{Pd}, \mathrm{Pt}, \mathrm{Zn}, \mathrm{Ga}$ ) were tested alone and in combination with graphene matrices acting as metal ion carriers. The antibacterial action of these systems was evaluated against antibiotic susceptible and antibiotic resistant strains of Acinetobacter baumannii, Staphylococcus aureus, Klebsiella pneumoniae and Pseudomonas aeruginosa, demonstrating the greatest activity with $\mathrm{Au}, \mathrm{Pd}$ and $\mathrm{Pt}$.

In order to develop eco-friendly materials with wide-spectrum antimicrobial activity for possible use in biomedical and food packaging applications, the work presented in [6] investigated the combination of two antibacterial agents-aluminum doped zinc oxide (Al-doped $\mathrm{ZnO}$, abbr. AZO) and silver (Ag) - in nanostructured layers deposited by different methods onto bioplastic polylactide (PLA) films. The two active agents showed preferential antibacterial activity against Gram-positive Staphylococcus aureus and Gramnegative Escherichia coli species, respectively, so that their synergistic dual action in the combined coatings was able to provide a total bacterial suppression against both species.

Coatings based on naturally occurring antimicrobial peptides (AMPs) were tested in [7] to produce antibacterial surfaces for biomaterials. Different AMPs (LL37, Magainin 2 , and Parasin 1) were covalently grafted onto an aldehyde plasma polymer (ALDpp), acting as a polymer interlayer that can be deposited onto different surfaces. Considerable reduction in bacterial colonization was demonstrated with Staphylococcus epidermidis, Staphylococcus aureus and Escherichia coli, while no significant cytotoxicity was found to primary human fibroblasts.

Another chemical-based route was employed in [8] to synthesize PLA-based amphiphilic copolymer micelles loaded with a photosensitizer (PS) of the BODIPY dyes family. Such micelles were sprayed on glass substrates to form a coating able to release BODIPY, resulting in an antibacterial action against Staphylococcus aureus.

In another work [9], the combination of the electrospinning method and the physical sputtering deposition technique was successfully used to produce antibacterial functionalized fiber scaffolds. In particular, Ag nanoparticles were sputter-deposited on electrospun polycaprolactone (PCL) fiber mats, without any significant damage induced on the soft polymer fibers, allowing the conferment of excellent antibacterial activity against Escherichia coli. The easy and flexible fabrication of these PCL-Ag mats can be applicable to several sectors, such as biomedical devices, bioremediation and antifouling systems in filtration, personal protective equipment (PPE), food packaging, etc.

Finally, a review [10] was presented about the use of hybrid organic-inorganic composites made by the incorporation of titanium dioxide $\left(\mathrm{TiO}_{2}\right)$ nanoparticles in protein-based materials. The characteristics of such compounds, including their antimicrobial properties, were discussed for possible usages in various applications (packaging, biomedical, pharmaceutical, environmental remediation, textiles, etc.), together with considerations on the issues related to $\mathrm{TiO}_{2}$ concentrations and need for standardization in production protocols.

As shown by the various works published in this Special Issue, it is clear how research efforts are continuously conducted by means of different strategies to provide antibacterial properties to surfaces for various applications. Many different materials are proposed, including organic and inorganic ones, as well as hybrid blends. Additionally, many different fabrication techniques can be employed, such as physical-based or chemical-based routes and their combination. At the same time, many aspects would require further examination, for example, to deeply assess the safety to human beings, the stability of the materials, and the duration of the antimicrobial activity. 
Acknowledgments: D.V. would like to thank all the Authors for their valuable contributions to this Special Issue, the Reviewers for their reviews and useful comments allowing the improvement of the submitted papers, and the Journal Editors for their kind support throughout the production of this Special Issue.

Conflicts of Interest: The author declares no conflict of interest.

\section{References}

1. Balos, S.; Puskar, T.; Potran, M.; Milekic, B.; Djurovic Koprivica, D.; Laban Terzija, J.; Gusic, I. Modulus, Strength and Cytotoxicity of PMMA-Silica Nanocomposites. Coatings 2020, 10, 583. [CrossRef]

2. Humayun, A.; Luo, Y.; Mills, D.K. Electrophoretic Deposition of Gentamicin-Loaded ZnHNTs-Chitosan on Titanium. Coatings 2020, 10, 944. [CrossRef]

3. Kvitek, O.; Mutylo, E.; Vokata, B.; Ulbrich, P.; Fajstavr, D.; Reznickova, A.; Svorcik, V. Photochemical Preparation of Silver Colloids in Hydroxypropyl Methylcellulose for Antibacterial Materials with Controlled Release of Silver. Coatings 2020, $10,1046$. [CrossRef]

4. Husain, F.M.; Hasan, I.; Qais, F.A.; Khan, R.A.; Alam, P.; Alsalme, A. Fabrication of Zinc Oxide-Xanthan Gum Nanocomposite via Green Route: Attenuation of Quorum Sensing Regulated Virulence Functions and Mitigation of Biofilm in Gram-Negative Bacterial Pathogens. Coatings 2020, 10, 1190. [CrossRef]

5. Slate, A.J.; Karaky, N.; Crowther, G.S.; Butler, J.A.; Banks, C.E.; McBain, A.J.; Whitehead, K.A. Graphene Matrices as Carriers for Metal Ions against Antibiotic Susceptible and Resistant Bacterial Pathogens. Coatings 2021, 11, 352. [CrossRef]

6. Valerini, D.; Tammaro, L.; Vigliotta, G.; Picariello, E.; Banfi, F.; Cavaliere, E.; Ciambriello, L.; Gavioli, L. Ag Functionalization of Al-Doped ZnO Nanostructured Coatings on PLA Substrate for Antibacterial Applications. Coatings 2020, 10, 1238. [CrossRef]

7. Griesser, S.S.; Jasieniak, M.; Vasilev, K.; Griesser, H.J. Antimicrobial Peptides Grafted onto a Plasma Polymer Interlayer Platform: Performance upon Extended Bacterial Challenge. Coatings 2021, 11, 68. [CrossRef]

8. Caruso, E.; Orlandi, V.T.; Malacarne, M.C.; Martegani, E.; Scanferla, C.; Pappalardo, D.; Vigliotta, G.; Izzo, L. Bodipy-Loaded Micelles Based on Polylactide as Surface Coating for Photodynamic Control of Staphylococcus aureus. Coatings 2021, 11, 223. [CrossRef]

9. Valerini, D.; Tammaro, L.; Vitali, R.; Guillot, G.; Rinaldi, A. Sputter-Deposited Ag Nanoparticles on Electrospun PCL Scaffolds: Morphology, Wettability and Antibacterial Activity. Coatings 2021, 11, 345. [CrossRef]

10. Anaya-Esparza, L.M.; Villagrán-de la Mora, Z.; Rodríguez-Barajas, N.; Sandoval-Contreras, T.; Nuño, K.; López-de la Mora, D.A.; Pérez-Larios, A.; Montalvo-González, E. Protein- $\mathrm{TiO}_{2}$ : A Functional Hybrid Composite with Diversified Applications. Coatings 2020, 10, 1194. [CrossRef] 